\title{
Degradación térmica de nanocomposites TEOS/resol y $\gamma$-APS/resol
}

\author{
D. SORIANO, A. MAZO, J. RUBIO, F. RUBIO, J. L. OTEO \\ Departamento de Química-Física de Superficies y Procesos, Instituto de Cerámica y Vidrio \\ (C.S.I.C.) - c/Kelsen, n 5. Campus Cantoblanco. 28049. Madrid, España.
}

\begin{abstract}
En este trabajo se ha estudiado la degradación térmica de nanocomposites resol-alcóxido (TEOS o APS) preparados mediante el proceso sol-gel. Esta degradación se ha caracterizado tanto mediante estudios cinéticos (Energía de Activación, orden de reacción y factor pre-exponencial) como por estudios degradativos (IPDT, IDT). En todos los casos la base de estudio han sido los termogramas realizados a distintas velocidades. Los valores de las energías de activación encontrados han sido similares a nanocomposites recogidos en la bibliografía (con valores comprendidos entre 100 y $200 \mathrm{~kJ} . \mathrm{mol}^{-1}$ ). Por otro lado, los órdenes de reacción han estado comprendidos entre 1,5 y 3,0 dependiendo del método utilizado y del nanocomposite analizado. Los valores de IPDT para todos los nanocomposites han sido superiores a $500{ }^{\circ} \mathrm{C}$ llegando en algunos casos a los $650{ }^{\circ} \mathrm{C}$. Finalmente se han calculado los valores del tiempo de vida media para una temperatura de $505{ }^{\circ} \mathrm{C}$ y una degradación del 95\% del nanocomposite, habiéndose alcanzado valores superiores a los 1100 minutos. Todos estos resultados se han correlacionado con los espectros IR en los que se ha observado que las mayores resistencias se alcanzan en aquellos nanocomposites en los que la resina interacciona con las partículas del alcóxido.
\end{abstract}

Palabras clave: Nanocomposites, resol, TEOS, APS, degradación térmica, cinética.

\section{Thermal degradation of TEOS/resol and $\gamma$-APS/resol nanocomposites}

The thermal degradation of TEOS-resol and APS-resol nanocomposites prepared by the sol-gel process has been studied in this work. Kinetic studies based on activation energies, reaction orders and pre-exponential factors as well as degradation analysis based on IPDT and IDT have been used in this work. For such studies thermograms at different heating velocities have been used. Activation energies between 100 and $200 \mathrm{~kJ} \cdot \mathrm{mol}^{-1}$ have been obtained in this work. Such values are of the same order as those found in the literature. On the other hand reaction orders vary between 1,5 and 3,0, depending on the nanocomposite analysed and the procedure used. At the same time IPDT values have been always higher than $500{ }^{\circ} \mathrm{C}$ reaching values close to $650{ }^{\circ} \mathrm{C}$ in some cases. Finally half life times calculated at a degradation temperature of $505^{\circ} \mathrm{C}$ and conversions of $95 \%$ have reached 1100 minutes for the best nanocomposites. All of these results have been correlated with IR spectra showing that those nanocomposites where resol molecules react with hydrolysed TEOS or APS they give the high degradation resistance.

Keywords: Nanocomposites, resol, TEOS, APS, thermal degradation, kinetics.

\section{INTRODUCCIÓN}

Las actuales demandas de materiales de altas prestaciones requieren el continuo desarrollo de nuevos composites que presenten una mayor resistencia a la oxidación y que, por lo tanto, se pueda prolongar el tiempo de vida útil de los composites utilizados hoy en día. En este sentido en los últimos años se ha llevado cabo toda una serie de modificaciones en los que podríamos denominar como composites clásicos, modificaciones que se han basado en la incorporación de nuevos tipos de rellenos inorgánicos tales como nanoparticulas esféricas y no esféricas, nanotubos y nanofibras de carbono, etc., así como también se han desarrollado composites con nuevas disposiciones tales como las multicapas, capas función gradiente, etc., todo ello, como ya se ha comentado, con el fin de mejorar el comportamiento térmico y mecánico de los composites (1-5).

La incorporación de nanopartículas inorgánicas en matrices clásicas utilizadas en los comúnmente denominados composites se ha llevado a cabo bien por adición de tales nanopartículas directamente a la resina (matriz del composite) o bien por medio de reacciones químicas entre la resina y moléculas de alcóxidos de silicio u otros alcóxidos metálicos. Este último procedimiento es el conocido como proceso solgel (6). Los materiales así conseguidos son los denominados 
nanocomposites puesto que el refuerzo se encuentra disperso en la matriz a escala nanométrica.

El procedimiento sol-gel es uno de los más utilizados hoy en día para la obtención de nanocomposites, lo que es debido tanto a la alta homogeneidad de las estructuras obtenidas así como a las elevadas áreas interfaciales puestas en juego (debido sobre todo a las nanopartículas formadas). Todo ello da lugar a que los nuevos materiales conocidos como nanocomposites posean excepcionales propiedades.

Cuando se obtienen nanocomposites entre nanopartículas y moléculas de resinas orgánicas se pueden dar dos tipos de interacciones entre las dos superficies puestas en juego. Por un lado se pueden dar enlaces químicos de carácter covalente como es el caso de los nanocomposites que utilizan TEOS (tetraetil ortosilicato), mientras que por otro lado se pueden dar interacciones del tipo de enlace de hidrógeno o bien quedar las nanopartículas embebidas en la reticulado formado por las moléculas de la resina, como es el caso de aquellos nanocomposites que utilizan poliimida (2).

El objetivo del presente trabajo ha sido el preparar nanocomposites a base de TEOS o $\gamma$-APS (gamma aminopropil trietoxisilano) y una resina fenólica tipo resol y estudiar la influencia de la concentración de ambos alcóxidos en la degradación térmica de los materiales obtenidos. En este estudio se comenzará describiendo someramente los distintos procedimientos y ecuaciones que existen para determinar los parámetros cinéticos de la degradación térmica, parámetros tales como la energía de activación, el orden de reacción y el factor pre-exponencial de Arrhenius, y también se comentará otro procedimiento que permite estimar la estabilidad térmica y el tiempo de vida media a partir de la curva termogravimétrica obtenida. Posteriormente se aplicarán tales procedimientos a las muestras de los nanocomposites preparados. Finalmente se discutirán los resultados obtenidos tanto en función del método empleado como en función del contenido y el tipo de alcóxido incorporado.

\section{EXPERIMENTAL}

\subsection{Reactivos y preparación de los nanocomposites}

Los reactivos utilizados en este trabajo han sido TEOS, (Merck), $\gamma$-APS (Gelest, GMBH) y resol (resina fenólica de Fers-Resins, S.A.), etanol (Merck), agua desionizada y $\mathrm{HCl}$ (35\%, Merck).

Los nanocomposites se prepararon mediante el proceso sol-gel para el cual se emplearon las siguientes relaciones molares: Etanol/Alcóxido: (4/1), agua/etanol: (5/1), y agua/ Alcóxido: $(20 / 1)$; en condiciones ácidas $(\mathrm{pH}=1)$. En primer lugar se llevó a cabo la reacción de hidrólisis del alcóxido durante 5 minutos a temperatura ambiente y en agitación mecánica. Transcurrido dicho tiempo se llevó a cabo la mezcla de la resina fenólica con distintas proporciones de la solución del alcóxido hidrolizado. Las proporciones en peso resol/alcóxido preparadas fueron: 100/0, 90/10, 80/20, 70/30, $60 / 40$ y 50/50. Después de un periodo de homogeneización se añadió el catalizador (CAT/C500 al $6 \%$ en peso respecto de la resina) y se mantuvo la reacción otros 10 minutos más en las mismas condiciones. Finalmente se eliminaron los disolventes durante 3 horas a $70{ }^{\circ} \mathrm{C}$. Durante este tiempo la resina curó (este tipo de resinas curan por debajo de $60^{\circ} \mathrm{C}$ ó a temperatura ambiente mediante la adición de una mezcla de ácidos catalizadores) y los nanocomposites obtenidos fueron piezas duras con la forma del recipiente de reacción. La nomenclatura utilizada para los nanocomposites fue la de utilizar la relación resol/alcóxido mencionada anteriormente anteponiendo las letras $\mathrm{R}$ para indicar resol y $\mathrm{T}$ o A para indicar TEOS o APS respectivamente.

\subsection{Equipos Experimentales}

Los estudios de estabilidad térmica de los nanocomposites preparados se realizaron mediante análisis termogravimétrico utilizando un equipo Perkin-Elmer (Pyris1-TGA) en atmósfera de aire y velocidades de calentamiento de 2,10 y $20^{\circ} \mathrm{C} / \mathrm{min}$. Las muestras se molieron previamente hasta un tamaño inferior a 100 micrómetros y se utilizaron unos $30 \mathrm{mg}$ en cada análisis.

Por otro lado, los nanocomposites preparados se estudiaron también por espectroscopia infrarroja utilizando un equipo FT-IR Perkin-Elmer (1760X). Se empleó la técnica de dilución de $\mathrm{KBr}(1 / 200 \mathrm{mg}$ de muestra/KBr, respectivamente). El intervalo de medida fue 4000-400 $\mathrm{cm}^{-1}$, la resolución fue de 2 $\mathrm{cm}^{-1}$ obteniéndose cada espectro como media de 10 medidas diferentes.

\section{ANÁLISIS CINÉTICO}

\subsection{Cinética de termodegradación.}

La ecuación general de la velocidad de degradación de polímeros y resinas viene definida por la Ec [1]:

$$
r=\frac{d \alpha}{d t}=k(T)^{*} f(\alpha)
$$

Donde $\mathrm{k}(\mathrm{T})$ es la constante de velocidad (tipo Arrhenius), $\mathrm{f}(\alpha)$ es una función que depende del mecanismo de degradación térmica de cada material, y $\alpha$ es el grado de conversión a una temperatura determinada. Para una velocidad de calentamiento no constante ó dinámica, $\beta\left({ }^{\circ} \mathrm{C} / \mathrm{min}\right)$, la ecuación [1] toma la forma general de la ecuación [2]:

$$
r=\frac{d \alpha}{d T} * \frac{d T}{d t}=\frac{d \alpha}{d T} * \beta=Z * e^{-E / R T *} f(\alpha)
$$

En donde $\mathrm{Z}\left(\mathrm{min}^{-1}\right)$ representa el factor pre-exponencial de la ecuación de Arrhenius, E $(\mathrm{kJ} / \mathrm{mol})$ la energía de activación del proceso, $\mathrm{R}$ y $\mathrm{T}$ la constante de los gases ideales y la temperatura, respectivamente. La resolución de la ecuación [2] utilizando las curvas termogravimétricas se puede llevar a cabo mediante dos tipos de procedimientos: diferenciales e integrales. En los primeros se asume que la forma de la función $\mathrm{f}(\alpha)$ es $(1-\alpha)^{\mathrm{n}}$, siendo $\mathrm{n}$ el orden de reacción. Por otro lado, en los segundos se resuelve la integral $\mathrm{g}(\alpha)$, la cual se obtiene despejando $f(\alpha)$ en la ecuación [2] tal y como se muestra en la ecuación [3]:

$$
g(\alpha)=\int \frac{d \alpha}{f(\alpha)}
$$

En el apartado siguiente se exponen los métodos 
más utilizados dentro de cada uno de estos dos grupos o procedimientos (diferencial o integral) para poder determinar la cinética de la degradación térmica de los nanocomposites preparados.

\subsubsection{Métodos diferenciales}

Los métodos diferenciales más utilizados para analizar la cinética de degradación de composites y nanocomposites han sido los de Kissinger, Freeman-Carroll, Friedman, Chang, Park y col., y Liu-Fan (7). A continuación se comentan brevemente los de Kissinger y Park y col., ya que serán los utilizados en este trabajo.

\section{Método de Kissinger}

De acuerdo con el comportamiento general de degradación de polímeros en el que $\mathrm{f}(\alpha)=(1-\alpha)^{\mathrm{n}}$, derivando y reorganizando la Ec [1] Kissinger (3) obtuvo la ecuación [4]:

$$
\frac{d \alpha}{d T}=\frac{Z}{\beta} * e^{-E / R T}(1-\alpha)^{n} \Rightarrow \ln \left(\frac{\beta}{T_{\max }^{2}}\right)=\ln \left(\frac{Z R}{E} n\left(1-\alpha_{\max }\right)^{(n-1)}\right)-\frac{E}{R T_{\max }}
$$

En esta ecuación $\mathrm{T}_{\max }$ es la temperatura correspondiente al pico del termograma. Si además se realiza la aproximación $\mathrm{n}(1-\alpha \mathrm{p})^{(\mathrm{n}-1)} \approx 1$, la expresión anterior conduce a la ecuación final [5], cuya representación gráfica permite obtener la energía de activación del valor de la pendiente y el factor pre-exponencial de la ordenada en el origen para las distintas velocidades de calentamiento empleadas.

$$
\ln \left(\frac{\beta}{T_{\max }^{2}}\right)=\ln \left(\frac{Z R}{E}\right)-\frac{E}{R T_{\max }}
$$

Por otro lado, Kissinger también propuso un método para calcular el orden de reacción, método que posteriormente fue expresado en forma matemática por Liu y Fan (8). Este método consiste en analizar la derivada primera del termograma y calcular la distancia al centro de gravedad desde los lados izquierdo y derecho cuando dicha curva se hace cero. No obstante como se acaba de comentar Liu y Fan propusieron un método matemático para realizar tal cálculo del orden de reacción, método expresado por la ecuación [6]. Es de tener en cuenta que la realización de la derivada puede ser también respecto al tiempo, si bien en este caso la relación señal/ruido es mayor (7).

$$
n=1.88 *\left[\frac{\left|d^{2} \alpha / d T^{2}\right|_{\text {izquierda }}}{\left|d^{2} \alpha / d T^{2}\right|_{\text {derecha }}}\right]
$$

\section{Método de Park y col.}

El método propuesto por Park y col., (6) introduce la dependencia del factor pre-exponencial con la temperatura quedando la ecuación final siguiente:

$$
\ln \beta=\ln Z_{0}+\frac{3}{2} \ln T_{\max }-\ln \left(\frac{E}{R T_{\max }}+\frac{1}{2}\right)-\frac{E}{R T}
$$

en donde el factor pre-exponencial es ahora $Z=Z_{o} T^{1 / 2}$

[7]

En este mismo sentido, la variación del orden de reacción con el grado de conversión, y su valor promedio, vendrán definidas por las expresiones matemáticas [8] y [9], respectivamente. Por otro lado utilizando las ecuaciones [10] y [11] se calcula la energía de activación y el correspondiente valor promedio, respectivamente.

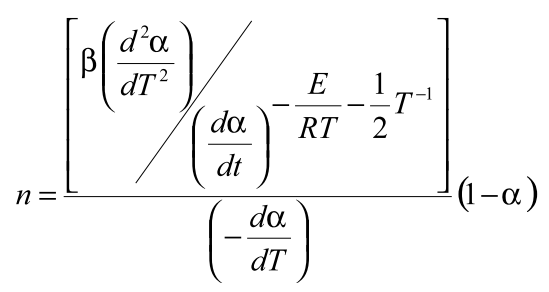

$$
n_{\text {promedio }}=\frac{\sum_{i=1}^{N} n_{i}\left(\alpha_{i}-\alpha_{i-1}\right)}{\alpha_{f}}
$$

$$
\begin{aligned}
& E=R T \ln \left[\frac{\left(\frac{d \alpha}{d t}\right)}{A o T^{1 / 2}(1-\alpha)^{n}}\right] \\
& E_{\text {promedio }}=\frac{\sum_{i=1}^{N} E_{i}\left(\alpha_{i}-\alpha_{i-1}\right)}{\alpha_{f}}
\end{aligned}
$$

\subsubsection{Métodos integrales.}

Los métodos integrales más utilizados para analizar la cinética de degradación de composites y nanocomposites han sido los de Horowitz-Metzger, Coats-Redfern y Ozawa. A continuación se comenta el de Ozawa por ser el más utilizado en la bibliografía y el que será empleado en este trabajo.

\section{Método de Flynn-Wall- Ozawa}

El método propuesto por Flynn y Wall, modificado por Ozawa (4) es uno de los más utilizados junto con el método diferencial de Kissinger. Dicho método agrupa e integra la ecuación [2] a partir de un desarrollo en serie de la misma. Posteriormente aproxima los dos primeros términos, según el modelo matemático de Doyle (9) en el cual se asume que la cinética de degradación se cumple para $0,11<\alpha<0,26$, obteniéndose así la ecuación final [12]. Además, en este modelo se considera que tanto la Energía de Activación como el factor pre-exponencial son independientes de la temperatura.

$$
\log \beta=-2.315+\log \left(\frac{Z E}{R}\right)-\log (g(\alpha))-0.4567 * \frac{E}{R} * \frac{1}{T}
$$




\subsubsection{Tiempo de vida media isotérmica}

Los métodos anteriores permiten determinar no solo E sino también el factor pre-exponencial, $\mathrm{Z}$, y el orden de reacción, $n$. Mediante estas tres variables cinéticas se puede calcular el tiempo de vida media isotérmica, $\tau$, a partir de la ecuación [13] (10).

$$
\ln \tau=\ln \left[\frac{\left[1-(1-\alpha)^{(1-n)}\right]}{Z(1-n)}\right]+\frac{E}{R T}
$$

Este tiempo de vida media es una indicación del tiempo que duraría un material sometido a una temperatura determinada.

\section{ESTABILIDAD TÉRMICA. PROCEDIMIENTO INTEGRAL DE DESCOMPOSICIÓN TÉRMICA (IPDT)}

Además del análisis cinético del proceso de degradación, existe otro procedimiento que permite determinar la estabilidad térmica tanto de polímeros como de composites el cual se basa en el análisis de las áreas encerradas por la curva termogravimétrica. Este procedimiento fue propuesto por Doyle (9) y permite obtener resultados con muy bajo error ya que no está influido por la velocidad de calentamiento ni por la cantidad de muestra utilizada. Dicho procedimiento es conocido como IPDT (integral procedural decomposition temperature) y, como se acaba de comentar, presenta una mayor fiabilidad que el procedimiento basado en la temperatura inicial de descomposición o IDT también desarrollado por Doyle.

El valor de IPDT viene definido por la ecuación [14];

$$
\operatorname{IPDT}\left({ }^{\circ} C\right)=A^{*} K^{*}\left(T_{f}-T_{i}\right)+T_{i}
$$

Siendo Ti y Tf las temperaturas inicial y final del análisis térmico. Las variables A y $\mathrm{K}$ se calculan mediante las ecuaciones [15] y [16]. Los valores de S1, S2 y S3 se calculan a partir de las áreas del registro termogravimétrico. En el apartado correspondiente se mostrará como se calculan dichos valores.

$$
\begin{aligned}
& K=\frac{(S 1+S 2)}{S 1} \\
& A=\frac{(S 1+S 2)}{(S 1+S 2+S 3)}
\end{aligned}
$$

\section{RESULTADOS}

\subsection{Espectroscopía Infrarroja}

En las Figuras 1 y 2 se muestran los espectros FT-IR obtenidos para los distintos nanocomposites preparados con resol-TEOS o resol-APS, respectivamente. En cada Figura se recoge también el espectro del alcóxido (TEOS o APS) hidrolizado y secado en las mismas condiciones experimentales pero sin ser añadido a la resina. El espectro correspondiente a la resina muestra las bandas típicas de una resina tipo resol. En dicho espectro las bandas que aparecen a 1510, 1500, 1475 y $1434 \mathrm{~cm}^{-1}$ corresponden a los puentes metileno situados en distintas posiciones o-p`, p-p`y o-o` (11); las bandas de muy pequeña intensidad situadas a 1360 y $1350 \mathrm{~cm}^{-1}$ corresponden a la deformación en el plano de los enlaces $-\mathrm{OH}$ del fenol (12); las bandas situadas a 1250 y $1200 \mathrm{~cm}^{-1}$ se asignan a la tensión $\mathrm{C}-\mathrm{OH}$ del fenol (11) si bien en este caso están desplazadas ligeramente hacia menores frecuencias (unos 20 $\mathrm{cm}^{-1}$ ) que las referenciadas en la bibliografía; a 1090 aparece una banda originada por los enlaces C-O-C de los puentes de oxidimetileno que unen los grupos fenol (11), banda que es típica de las resinas resol y que sirve para diferenciarlas de las novolacas; en dicha banda aparece un hombro a mayor frecuencia $\left(1115 \mathrm{~cm}^{-1}\right)$ la correspondiente a la deformación en el plano de los enlaces $\mathrm{CH}$ en sustitución 1,2,4 aromáticos. A 870, 812 y $760 \mathrm{~cm}^{-1}$ aparecen las vibraciones de flexión C-H fuera del plano del anillo cuando el anillo posee sustituciones 1,2 , y 1,2,6, o 1,4 y 1,2,4, o 1,2,4, respectivamente $(11,12)$. A frecuencias inferiores a $700 \mathrm{~cm}^{-1}$ las bandas que aparecen son de pequeña intensidad y corresponden a deformaciones de los distintos enlaces ya mencionados $(2,12)$.

Por otro lado, el espectro del TEOS hidrolizado (Figura 1) presenta una banda intensa situada a sobre los $1080 \mathrm{~cm}^{-1}$ la cual posee un hombro también intenso sobre los $1200 \mathrm{~cm}^{-1}$, siendo la primera originada por tensiones simétricas y la segunda por tensiones asimétricas de enlaces Si-O-Si (13). A $950 \mathrm{~cm}^{-1}$ aparece una nueva banda asignada a enlaces $\mathrm{Si}-\mathrm{OH}$, y a 795 $\mathrm{cm}^{-1}$ también aparece otra originada por el tetraedro $\mathrm{SiO}^{4-}$, banda que se desplaza hasta los $800 \mathrm{~cm}^{-1}$ si la unión es del tipo Si-O-Si; y finalmente a $460 \mathrm{~cm}^{-1}$ aparece la banda de flexión de los enlaces O-Si-O (13) siempre que la red sea de sílice, si bien a medida que la red es más orgánica dicha banda se desplaza a menores frecuencias, pudiendo estar por debajo de los $400 \mathrm{~cm}^{-1}$ como ocurre con los polisiloxanos (13). Diferentes trabajos han mostrado que el TEOS una vez hidrolizado en medio ácido la lugar a reacciones de autocondensación que pueden transcurrir formando una estructura global con entrecruzamiento en las tres dimensiones (tridimensional) o bien puede formar cadenas lineales (14). Ambas estructuras, tridimensional o lineal, originan dos bandas infrarrojas a 1200 y $1150 \mathrm{~cm}^{-1}$, respectivamente, las cuales se encuentran englobadas en la hombro, ya comentado, que se observa a $1200 \mathrm{~cm}^{-1}$. Hay que tener en cuenta que el espectro del TEOS hidrolizado y policondensado se ha obtenido para TEOS puro, es decir en ausencia de resina, y por ello las bandas anteriores pueden cambiar ligeramente cuando el TEOS se encuentre en el medio orgánico de la resina resol y ambos comiencen a condensar/reticular para formar el nanocomposite.

Finalmente el APS hidrolizado presenta las mismas bandas mencionadas para el TEOS hidrolizado y algunas más debido a los enlaces Si-C y C-N (15). Cabe destacar dos aspectos importantes en este caso, el primero es que el APS no forma una clara estructura tridimensional ya que no puede condensar por uno de los enlaces (el correspondiente al grupo aminopropílico) por lo que la banda situada a $1200 \mathrm{~cm}^{-1}$ (la cual aquí aparece a $1190 \mathrm{~cm}^{-1}$ ) es de muy baja intensidad, y por lo tanto su polimerización es mas de tipo lineal por lo que la banda situada a $1150 \mathrm{~cm}^{-1}$ es ahora más intensa y aparece a frecuencias algo más bajas (sobre los $1120 \mathrm{~cm}^{-1}$ ), tal y como se puede observar en la Figura 2. Esto se corrobora también por la banda que aparece sobre los $1025 \mathrm{~cm}^{-1}$ la cual corresponde a 
enlaces $\mathrm{Si}-\mathrm{O}-\mathrm{Si}(\mathrm{CH})^{2-}$, típica de siliconas (13). Por otro lado, la policondensación del APS hidrolizado origina enlaces del tipo O-Si-C que dan lugar a una banda situada sobre los $860 \mathrm{~cm}^{-1}$ (13). Igualmente que lo comentado para el caso del TEOS, este espectro corresponde al APS hidrolizado y policondensado en ausencia de resina, y por lo tanto dichas bandas pueden cambiar cuando exista resol.

La incorporación de TEOS o APS hidrolizados en la estructura de la resina resol produce ciertos cambios en las bandas IR debido a que ambos compuestos pueden quedar embebidos en la resina o bien formar enlaces químicos con la misma (Figuras 1 y 2). En los nanocomposites resol-TEOS (Figura 1) se observa que la incorporación de hasta un $40 \%$ de TEOS produce un cambio importante en la relación de las bandas situadas a 1200 y $1080 \mathrm{~cm}^{-1}$ ya que para el resol ambas bandas poseen intensidades similares, mientras que en los nanocomposites la situada a $1080 \mathrm{~cm}^{-1}$ es más intensa. Este resultado es debido a que el TEOS una vez hidrolizado está dando lugar a enlaces del tipo Si-O-Si los cuales a su vez forman partículas primarias de sílice dentro de la estructura de la resina. Esto se corrobora por el desplazamiento de la banda de $750 \mathrm{~cm}^{-1}$ hasta los $795 \mathrm{~cm}^{-1}$ a la vez que comienza a aparecer una banda sobre los $400 \mathrm{~cm}^{-1}$ que se desplaza hasta los $462 \mathrm{~cm}^{-1}$. Sin embargo para concentraciones del 50 y $60 \%$ de TEOS la relación de las bandas 1200 y $1080 \mathrm{~cm}^{-1}$ cambia, siendo ahora más intensa la de mayor frecuencia a la vez que aumenta también en achura, resultado que puede ser debido a dos contribuciones: la primera sería una indicación de que se está formando una estructura inorgánica tridimensional (responsable de la banda situada a $1200 \mathrm{~cm}^{-1}$, como se ha comentado más arriba) de sílice en la que se encontraría el resol, y la segunda sería que el TEOS hidrolizado rompe o impide la formación de enlaces C-O-C de la resina y forma o mantiene los grupos fenol (que originan la banda situada a $1200 \mathrm{~cm}^{-1}$ ). Aunque ambas posibilidades son factibles, sin embargo es mas probable la primera ya que si los grupos fenol estuvieran más libres y no formando parte de la resina estos darían lugar a bandas situadas por encima de $1200 \mathrm{~cm}^{-1}$ (sobre 1220 y $1270 \mathrm{~cm}^{-1}$ ), tal y como ahora se comentará para los nanocomposites resol-APS.

En los nanocomposites resol-APS (Figura 2) se observa un cambio importante desde las primeras incorporaciones de APS. Así la relación de las bandas situadas a 1200 y $1080 \mathrm{~cm}^{-1}$ cambia radicalmente, siendo ahora más intensa la de mayor frecuencia y apareciendo ésta sobre los $1211 \mathrm{~cm}^{-1}$ y con una anchura bastante mayor a la que posee el resol solo. Como la banda situada a $1200 \mathrm{~cm}^{-1}$ corresponde al grupo fenol y la de 1080 a los puentes $\mathrm{C}-\mathrm{O}-\mathrm{C}$, el resultado encontrado indica que el APS reacciona con los enlaces C-O-C que mantienen unidos a los grupos fenol y por lo tanto no deja que condense la resina de acuerdo a su mecanismo de polimerización (16). Por otro lado, el aumento en intensidad de la banda situada sobre los $1200 \mathrm{~cm}^{-1}$ podría indicar la formación de nuevos grupos fenol, sin embargo esta banda aumenta también en anchura y en posición (llegando a alcanzar los $1230 \mathrm{~cm}^{-1}$ para el nanocomposite de mayor contenido en APS), lo que indica que los grupos fenol deben estar formando parte tanto de estructuras en las que se encuentren bastante libres (y por ello las bandas aparecen a mayor frecuencia) como en estructuras muy desordenadas (y por ello las bandas se ensanchan). A esto hay que añadir también que en los nanocomposites de mayor contenido en APS se aprecia un hombro sobre los 1200 $\mathrm{cm}^{-1}$ que obviamente debe estar originado por los grupos fenol situados en zonas (nanozonas) de resina pura, mientras que el resto deben estar formando el nanocomposite con el APS. Además se observa como al aumentar el contenido en APS aumentan en intensidad las bandas situadas sobre los $1025 \mathrm{y}$ $1080 \mathrm{~cm}^{-1}$, lo que corresponde con un aumento del contenido en APS en el nanocomposite. A su vez se aprecia como la banda situada sobre los $1080 \mathrm{~cm}^{-1}$ se desplaza hasta los 1115 $\mathrm{cm}^{-1}$, indicando que la estructura de la red de sílice que forma el APS es más de tipo lineal que cíclica, ya que la banda situada a $1200 \mathrm{~cm}^{-1}$ no cambia. En resumen, en los nanocomposites preparados con APS se puede suponer la formación de una red híbrida con el resol, afirmación que se corrobora por la forma de la banda situada a $1435 \mathrm{~cm}^{-1}$ que es originada por las vibraciones tijera de enlaces $\mathrm{C}-\mathrm{H}_{2}$ alifáticos, dicha banda se ensancha y se convierte en un hombro a medida que aumenta la concentración de APS lo que indica que este compuesto está interaccionando con tales enlaces.

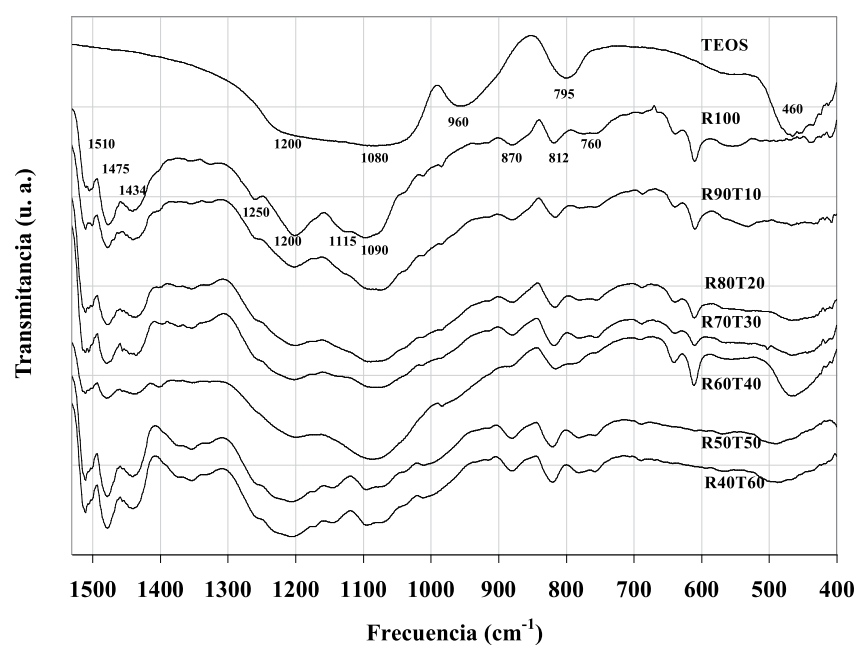

Fig. 1- Espectros IR de nanocomposites resol/TEOS.

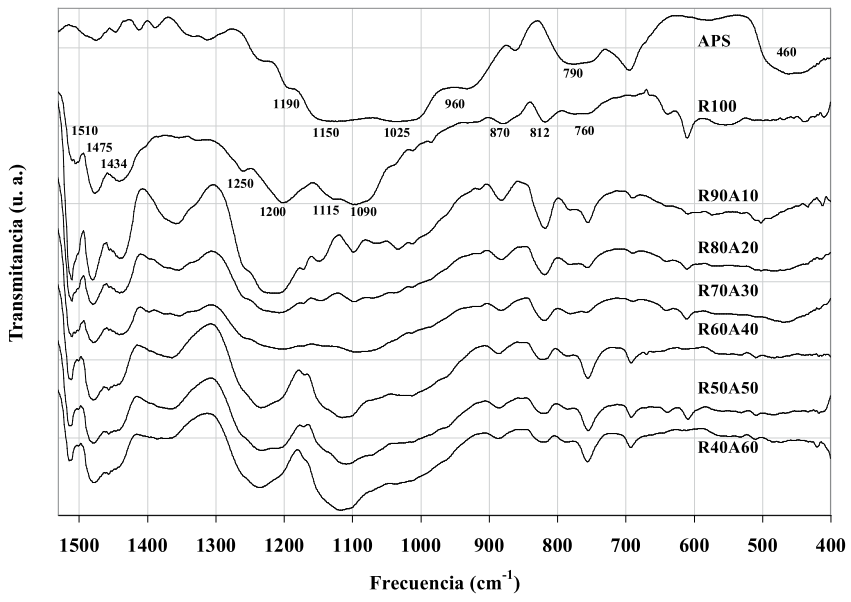

Fig. 2- Espectros IR de nanocomposites resol/APS

\subsection{Análisis Térmico}

En la Figura 3 se muestran los termogramas de los nanocomposites preparados a partir de resol y TEOS para una misma velocidad de calentamiento, mientras que la Figura 4 muestra dichos termogramas a distintas velocidades para uno de resol/APS. En todas las curvas se observa una 
primera pérdida de peso que transcurre hasta unos $200^{\circ} \mathrm{C}$ y que corresponde a la eliminación de volátiles presentes en las muestras. Dichos volátiles provienen tanto de la propia reacción catalítica de la resina como de las reacciones de condensación de los alcóxidos hidrolizados utilizados. Así se puede determinar que esta primera pérdida de peso es menor para la resina pura $(3,5 \%)$ y aumenta con el contenido en alcóxido llegando hasta el 10,7\% o al 14,8\% cuando dicho contenido es del $60 \%$ en TEOS o en APS, respectivamente. La mayor pérdida de peso que tiene lugar con el APS se debe a la presencia de los radicales n-propil-amina que no se hidrolizan y quedan en la molécula de APS.

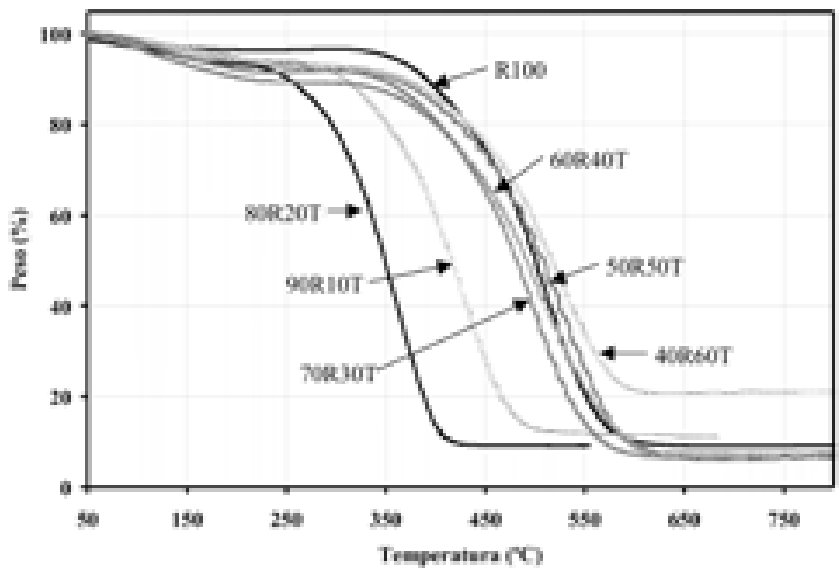

Fig. 3- Curvas termogravimétricas de nanocomposites resol/TEOS ( $\beta$ $\left.=10^{\circ} \mathrm{C} \cdot \mathrm{min}^{-1}\right)$.

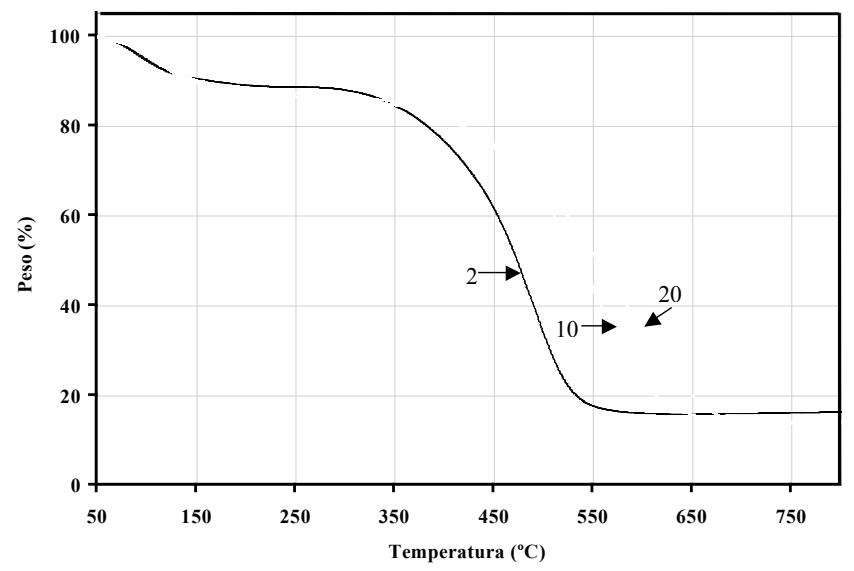

Fig. 4- Curvas termogravimétricas del nanocomposite resol/APS $(80 / 20)$ a diferentes velocidades de calentamiento. S1, S2 y S3: áreas para cálculo de IPDT.

A partir de estas curvas TG se ha procedido a calcular los distintos parámetros cinéticos y de estabilidad térmica de los distintos nanocomposites de acuerdo con los procedimientos mencionados en el Apartado anterior. A continuación se recogen y discuten los resultados obtenidos.

\subsection{1.- Estudio Cinético}

Métodos Diferenciales (Kissinger, Park y col.).

Las Figuras 5 y 6 muestran las representaciones de la ecuación de Kissinger para los distintos nanocomposites de
Resol/TEOS y Resol/APS, respectivamente. En todos los casos se han obtenido representaciones rectas con coeficientes de regresión superiores a 0,9 .

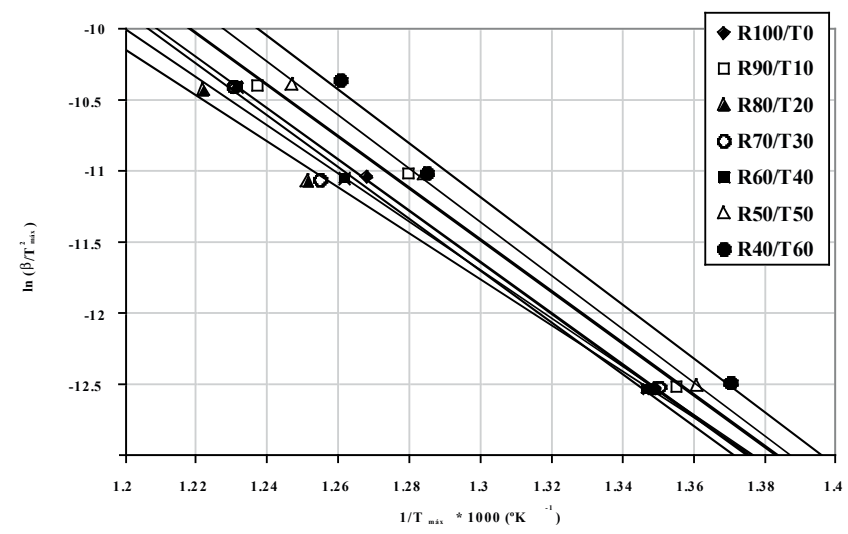

Fig. 5- Representación del método de Kissinger para nanocomposites resol/TEOS.

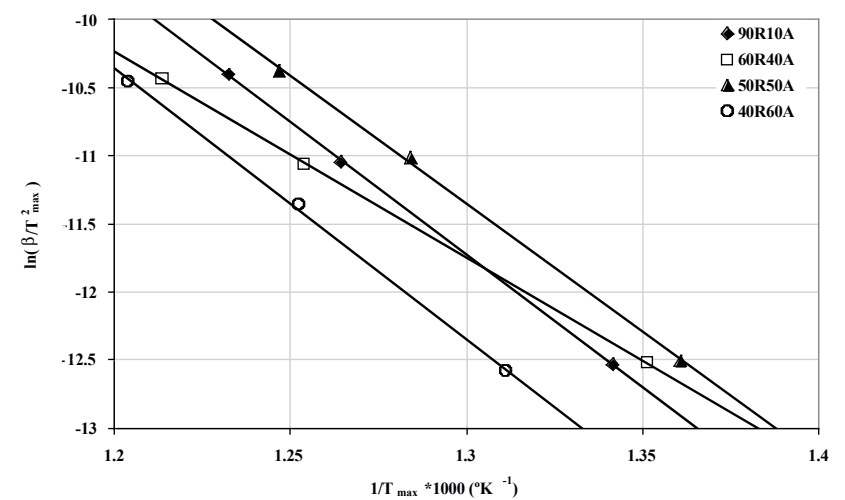

Fig. 6- Representación del método de Kissinger para nanocomposites resol/APS.

A partir de la pendiente de estas rectas se han obtenido los valores de E de acuerdo con la ecuación [5]. Y a partir de la variación de dicha pendiente con la velocidad de calentamiento se obtiene el factor pre-exponencial. Dichos valores se recogen en la Tabla 1.

Por otro lado, las Figuras 7 y 8 muestran las representaciones de la ecuación de Park y col., para los nanocomposites Resol/ TEOS y Resol/ APS, respectivamente. Tal y como se mencionó anteriormente este método permite determinar la evolución tanto de la energía de activación como del orden de reacción en todo el intervalo de conversión $\alpha$. Así se observa que E aumenta con $\alpha$ lo que se puede atribuir a que para bajas $\alpha$ los valores obtenidos corresponden a la degradación térmica de la resina mientras que para elevadas $\alpha$ los valores de E se corresponden más con la degradación de las moléculas de la resina unidas químicamente a las nanopartículas de TEOS o de APS. En este sentido los valores de E utilizados en la discusión de este trabajo corresponden a $\alpha=0,5$. Por otro lado se observa como la energía de activación también aumenta para el resol solo, resultado que puede interpretarse según el modelo de Chan y Balke (17) los cuales propusieron un mecanismo de degradación de polímeros orgánicos (en su caso polipropileno) de acuerdo a tres regiones o etapas diferentes. En la primera zona (0-10\% de conversión) consideraron un pseudo-mecanismo de primer orden, con energías próximas 
a $135 \mathrm{~kJ} / \mathrm{mol}$, degradación que atribuyeron a la destrucción mecánica de los enlaces débiles del polímero. La segunda región (a partir del 30\% de conversión) se caracterizaba por las grandes pérdidas de peso asociadas al elevado número de roturas de cadena; considerándola también como un pseudo-mecanismo de primer orden, con elevadas energías de activación $(330 \mathrm{~kJ} / \mathrm{mol})$. Y por último, una tercera zona de transición situada entre ambas asignada a la destrucción de cadenas de grafito entrecruzadas con el material polimérico, cuya energía de activación podría ser superior a los $330 \mathrm{~kJ} /$ mol. En nuestro caso para el resol la variación de E con $\alpha$ no es tan grande como la propuesta por Chan y Balke (17) aunque sí que se observa tal incremento.

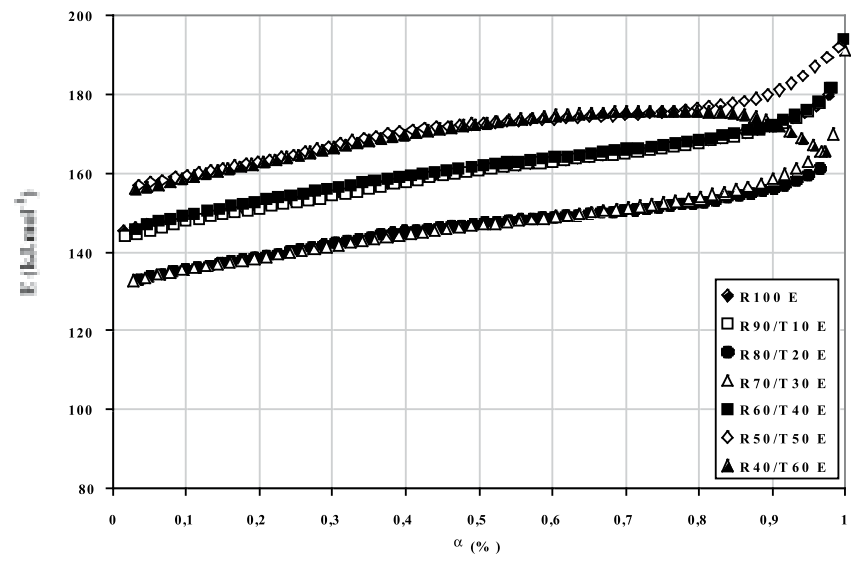

Fig. 7- Representación del método de Park para nanocomposites Resol/TEOS. $\beta=5^{\circ} \mathrm{C} \cdot \mathrm{min}^{-1}$.

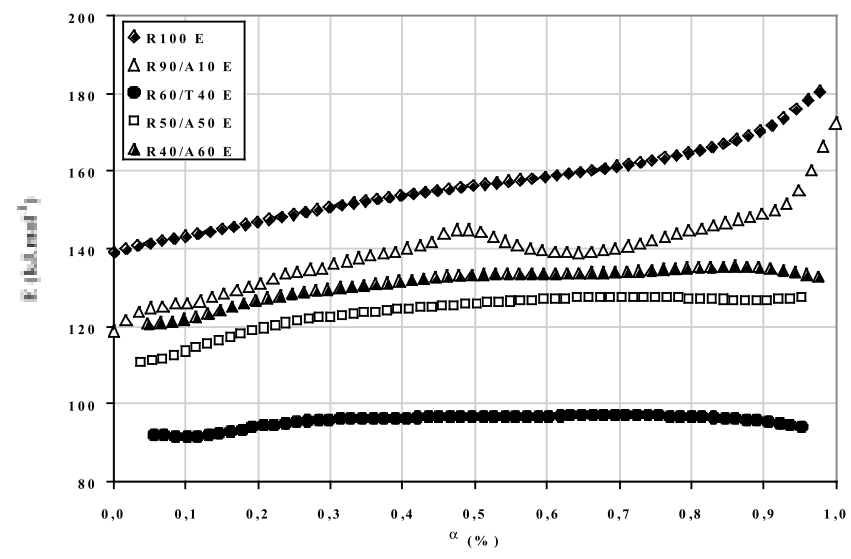

Fig. 8- Representación del método de Park para nanocomposites Resol $/$ APS. $\beta=5^{\circ} \mathrm{C} \cdot \mathrm{min}^{-1}$.

\section{Método Integral (Ozawa)}

Las Figuras 9 y 10 muestran las representaciones de la ecuación deOzawa paralos distintosnanocomposites obtenidos a partir de Resol/ TEOS y Resol / APS, respectivamente. En este método la representación se hace en función de la conversión y en teoría la misma debe ser recta hasta valores próximos al $20 \%$. Sin embargo en nuestro caso se han obtenido líneas rectas para todo el intervalo de conversiones. Las rectas obtenidas en estas representaciones han alcanzado coeficientes de regresión superiores a 0,95 .

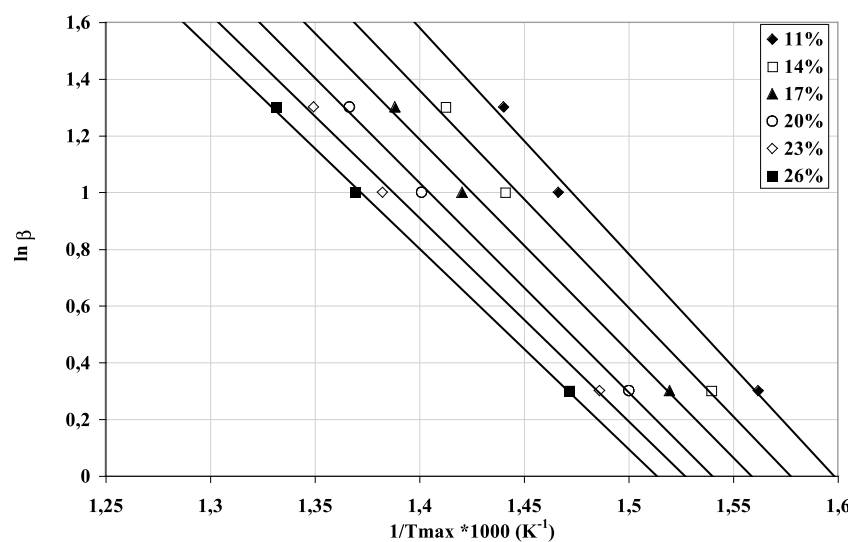

Fig. 9- Representación del método de Ozawa para el nanocomposite Resol/TEOS 80/20 a diferentes $\alpha . \beta=5{ }^{\circ} \mathrm{C} \cdot \mathrm{min}^{-1}$.

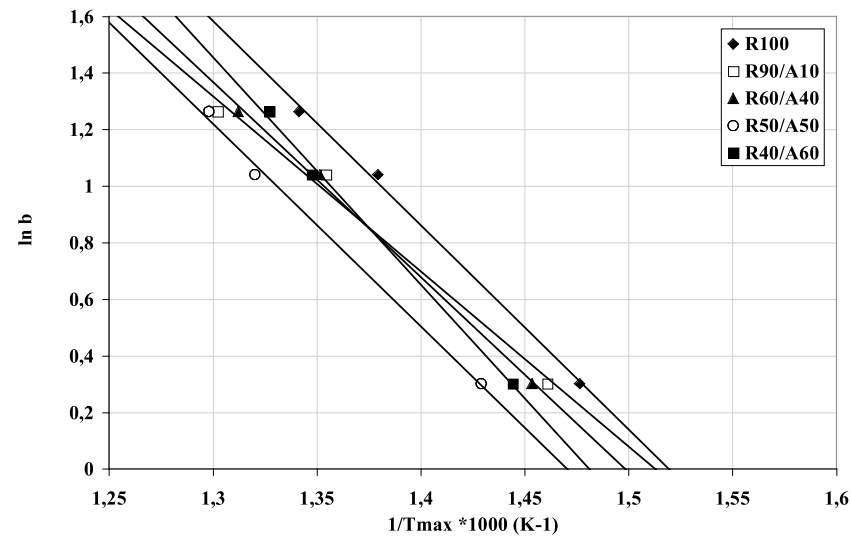

Fig. 10- Representación del método de Ozawa para nanocomposites Resol $/$ APS. $\alpha=0.5 . \beta=5^{\circ} \mathrm{C} \cdot \mathrm{min}^{-1}$.

De las pendientes de esas rectas se han obtenido los valores de E para cada nanocomposite. Dichos valores están recogidos en la Tabla 1.

TABLA I. CONSTANTES CINÉTICAS DE LA DEGRADACIÓN DE NANOCOMPOSITES RESOL/ALCÓXIDO

\begin{tabular}{|c|c|c|c|c|c|c|c|c|}
\hline \multirow{2}{*}{ Sistema } & \multirow{2}{*}{ Nanocomposites } & \multicolumn{3}{|c|}{$\mathrm{E}\left(\mathrm{kJ} \cdot \mathrm{mol}^{-1}\right)$} & \multicolumn{2}{|c|}{$\mathrm{n}$} & \multicolumn{2}{|c|}{$Z\left({ }^{*} 10^{9}\right)$} \\
\hline & & $\mathrm{K}$ & $\mathrm{P}$ & $\mathrm{O}$ & K & $\mathrm{P}$ & K & $\mathrm{P}$ \\
\hline Resol & R100 & 150,0 & 159,7 & 147,0 & 2,5 & 1,5 & 2,5 & 2,5 \\
\hline \multirow{6}{*}{$\begin{array}{l}\text { Resol- } \\
\text { TEOS }\end{array}$} & R90/T10 & 151,1 & 169,4 & 130,1 & 2,4 & 1,5 & 3,5 & 2,9 \\
\hline & $\mathrm{R} 80 / \mathrm{T} 20$ & 134,4 & 137,2 & 128,8 & 2,4 & 1,4 & 1,8 & 1,5 \\
\hline & $\mathrm{R} 70 / \mathrm{T} 30$ & 141,0 & 153,6 & 139,1 & 2,5 & 1,5 & 5,3 & 0,5 \\
\hline & $\mathrm{R} 60 / \mathrm{T} 40$ & 151,4 & 161,3 & 139,7 & 2,5 & 1,5 & 2,9 & 2,5 \\
\hline & $\mathrm{R} 50 / \mathrm{T} 50$ & 156,4 & 184,1 & 141,8 & 2,6 & 1,4 & 8,8 & 2,4 \\
\hline & $\mathrm{R} 40 / \mathrm{T} 60$ & 157,2 & 180,4 & 141,8 & 2,1 & 1,7 & 12,3 & 3,6 \\
\hline \multirow{6}{*}{$\begin{array}{l}\text { Resol } \\
\text { - APS }\end{array}$} & R90/A10 & 153,2 & 169,3 & 124,6 & 3,3 & 2,1 & 16,0 & 4,7 \\
\hline & R80/AT20 & 140,8 & 148,6 & 120,2 & 2,6 & 2,0 & 12,1 & 3,5 \\
\hline & R70/A30 & 129,3 & 139,2 & 126,3 & 2,2 & 2,2 & 3,6 & 1,8 \\
\hline & $\mathrm{R} 60 / \mathrm{A} 40$ & 126,0 & 137,2 & 131,0 & 1,9 & 2,3 & 0,04 & 0,01 \\
\hline & R50/A50 & 156,4 & 175,3 & 139,3 & 2,8 & 1,8 & 9,2 & 3,6 \\
\hline & $\mathrm{R} 40 / 60$ & 167,2 & 178,2 & 148,4 & 3,0 & 2,1 & 15,0 & 6,2 \\
\hline
\end{tabular}

* K: Kissinger; P: Park y col.; O: Ozawa 
Como puede verse en esta Tabla al comparar los distintos procedimientos (diferencial o integral, los valores obtenidos para E están bastante próximos entre sí. Para las distintas relaciones Resol/alcóxido, el método que muestra mayor variación en E es el de Park y col., si bien los valores no se separan demasiado de los de los otros dos métodos. En cuanto a la variación de E en función de la relación Resol/alcóxido se observa que tanto para los nanocomposites preparados con TEOS o con APS existe una disminución de la energía al aumentar el contenido en alcóxido hasta una relación que está comprendida entre el 20 y el $30 \%$ en alcóxido. Mayores concentraciones producen un aumento de E.

Por otro lado, en cuanto al orden de reacción y al factor pre-exponencial los valores obtenidos dependen en gran medida del método utilizado. En general el procedimiento de Kissinger proporciona valores superiores al de Park y col. Puede observarse que el orden de reacción no depende de la concentración de alcóxido empleada, aunque si que depende del tipo de alcóxido siendo mayor para los nanocomposites preparados con APS. Por el contrario el factor pre-exponencial sigue una variación parecida a E, alcanzado los mínimos valores para concentraciones de alcóxido comprendidas entre el 20 y el $40 \%$ en el nanocomposite. Los pequeños valores del factor pre-exponencial correspondientes al nanocomposite R60/ A40 coinciden con que dicha muestra presenta la menor energía de activación y menor orden de reacción.

\subsection{2.- Estabilidad Térmica. Degradación. IPDT}

Los valores de los IPDTs se han obtenido a partir de las curvas termogravimétricas utilizando las ecuaciones [14] a [16]. En todos los casos se ha utilizado la curva correspondiente a una velocidad de calentamiento de $10{ }^{\circ} \mathrm{C} \cdot \mathrm{min}^{-1}$. Los valores de S1, S2 y S3 se han calculado de acuerdo tal y como se muestra en la Figura 4. En la Tabla 2 se recogen los valores de IPDT, IDT y Tmax para cada nanocomposite.

De acuerdo a los resultados de la Tabla 2 la degradación térmica de los nanocomposites resol-alcóxido aumenta con el contenido en alcóxido hasta una concentración comprendida entre el 30 y el $40 \%$ de éste. Mayores concentraciones en alcóxido producen un aumento en la estabilidad térmica de los nanocomposites. Estos resultados están de acuerdo con los valores de la energía de activación recogidos en la Tabla 1. En cuanto la influencia del tipo de alcóxido (TEOS o APS) los datos de la Tabla 2 indican que prácticamente los dos ejercen la misma influencia respecto a la degradación térmica de los nanocomposites.

Por otro lado y tal y como se comentó en el apartado de Introducción, los valores de Tmax e IDT no son muy apropiados para caracterizar la degradación térmica de materiales pues como puede observarse en la Tabla 2 dichos valores oscilan muy poco de una muestra a otra y no siguen un orden coherente con el contenido en alcóxido.

De acuerdo a los resultados obtenidos en la utilización de los distintos procedimientos para calcular la degradación térmica de nanocomposites, se puede decir que el método de Park y col., resulta ser el más completo, ya que permite obtener los valores de la energía de activación y del orden de reacción en todo el intervalo de conversión, a la vez que también se puede calcular el valor del factor pre-exponencial. Si hasta la fecha los métodos de Kissinger y Ozawa eran los más utilizados debido a la facilidad de realizar cálculos, sin embargo hoy en día el método de Park y col., puede
TABLA II. VALORES DE $\mathrm{T}_{\mathrm{MAX}}$ IDT E IPDT DE NANOCOMPOSITES RESOL/ALCÓXIDO

\begin{tabular}{|c|c|c|c|c|}
\hline Sistema & Nanocomposite & $\mathrm{T}_{\max }(\mathrm{K})$ & IDT (K) & $\begin{array}{c}\text { IPDT } \\
(\mathrm{K})\end{array}$ \\
\hline Resol & $\mathrm{R} 100$ & 474 & 387 & 584,0 \\
\hline \multirow{4}{*}{ Resol - TEOS } & $\mathrm{R} 90 / \mathrm{T} 10$ & 467 & 379 & 602,6 \\
\cline { 2 - 5 } & $\mathrm{R} 80 / \mathrm{T} 20$ & 470 & 391 & 584,3 \\
\cline { 2 - 5 } & $\mathrm{R} 70 / \mathrm{T} 30$ & 469 & 383 & 557,9 \\
\cline { 2 - 5 } & $\mathrm{R} 60 / \mathrm{T} 40$ & 470 & 381 & 549,2 \\
\cline { 2 - 5 } & $\mathrm{R} 50 / \mathrm{T} 50$ & 464 & 370 & 610,5 \\
\cline { 2 - 5 } & $\mathrm{R} 40 / \mathrm{T} 60$ & 457 & 358 & 645,4 \\
\hline \multirow{5}{*}{ Resol - APS } & $\mathrm{R} 90 / \mathrm{A} 10$ & 506 & 323 & 510,4 \\
\cline { 2 - 5 } & $\mathrm{R} 80 / \mathrm{A} 20$ & 510 & 320 & 505,3 \\
\cline { 2 - 5 } & $\mathrm{R} 70 / \mathrm{A} 30$ & 510 & 321 & 531,7 \\
\cline { 2 - 5 } & $\mathrm{R} 60 / \mathrm{A} 40$ & 513 & 320 & 581,5 \\
\cline { 2 - 5 } & $\mathrm{R} 50 / \mathrm{A} 50$ & 534 & 310 & 652,8 \\
\cline { 2 - 5 } & $\mathrm{R} 40 / \mathrm{A} 60$ & 527 & 310 & 645,3 \\
\hline
\end{tabular}

considerarse tan rápido como los anteriores. Además, dicho método proporciona valores muy similares a los otros dos, lo que corrobora su viabilidad para la determinación de las constantes cinéticas de degradación.

Respecto a los resultados obtenidos de E para los nanocomposites, los valores son próximos a los obtenidos por otros autores. Así Lin y col. (2) aplicando diversos métodos calcularon los valores de E para la degradación en nitrógeno de híbridos resol/alcóxido con relación $80 / 20$ y obtuvieron

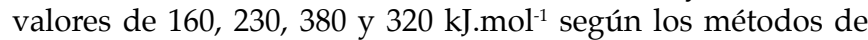
Kissinger, Friedman y Ozawa, respectivamente, mientras que Gao y col., (18) obtuvieron, para la degradación en aire de resinas fenólicas tipo resol, valores E alrededor de $110 \mathrm{~kJ} / \mathrm{mol}$. En nuestro estudio los resultados obtenidos (teniendo en cuenta que la degradación es en aire) para el nanocomposite $80 / 20$ han sido de 134,4, 137,2 y 128,8 kJ.mol ${ }^{-1}$ para los métodos de Kissinger, Park y col., y Ozawa, respectivamente. Es decir valores muy próximos a los comentados. Este resultado está en relación con el obtenido por Park (19) quien concluyó que con la incorporación de alcóxidos a compuestos de poliestireno reforzados con sílice se aumentaba la adhesión interfásica mejorando así la estabilidad térmica. Resultado que contradice al de Wang (20) el cual sostuvo que la energía de activación para un primer estadio de la degradación de híbridos poli amida-imida-sílice disminuía al aumentar el contenido inorgánico. Otros trabajos como el de Sengupta y col., (21) en el cual caracterizaron cinéticamente nanocomposites poliamida-TEOS incorporando el alcóxido mediante la técnica sol-gel, en él se demostró que para contenidos en alcóxido inferiores al 3\% se mejoraba la degradación térmica, pero en porcentajes comprendidos entre el 3 y el $10 \%$ ocurría el caso contrario. Luego el tipo de polímero y la concentración de alcóxido deben de tener una influencia fundamental a la hora de conseguir una mejorar de la estabilidad térmica.

En cuanto a la concentración de alcóxido en este trabajo se ha observado que la energía de activación disminuye con dicho contenido hasta alcanzar un mínimo para un $20 \%$ en TEOS. A partir de dicho contenido E aumenta al hacerlo el del alcóxido, si bien los valores no son mucho mayores a los de la resina sola. Por otro lado, el tipo de alcóxido (TEOS o APS) no da lugar a cambios significativos en E, a pesar de que los radicales orgánicos tipo amino, como los del APS, se suelen utilizar para catalizar resinas fenólicas y mejorar las propiedades de éstas.

Por otro lado cuando se estudia la degradación térmica de 
estos nanocomposites mediante los valores de IPDT se observa en general un aumento de la estabilidad con el contenido en alcóxido y en este sentido la temperatura de máxima degradación aumenta en los nanocomposites preparados con APS. Este resultado indica que la formación de cadenas lineales de sílice y el no romper los enlaces C-O-C de la resina por parte del APS favorece la estabilidad térmica. Diferentes estudios han demostrado que el IPDT de polímeros y resinas aumenta con el contenido en material inorgánico, ya se éste procedente de partículas o nanopartículas $(1,22)$.

Finalmente utilizando los valores de $\mathrm{E}, \mathrm{n}$ y $\mathrm{Z}$ se han calculado los tiempos de vida media de la degradación de los nanocomposites obtenidos. Para ello se ha utilizado la ecuación [13]. En la Figura 11 se muestra la evolución de tiempo de vida media a $505{ }^{\circ} \mathrm{C}$ para una degradación del $95 \%$ de los distintos nanocomposites. Se ha realizado un ajuste a un polinomio con el fin de mostrar solamente la tendencia. De nuevo se observan unos valores mínimos para concentraciones de alcóxidos comprendidas entre el 20 y el 30\%. Sin embargo para concentraciones superiores al $40 \%$ el tiempo de vida media es similar o superior al de la resina inicial. Por lo tanto se puede concluir que la incorporación de TEOS o APS en resina fenólica tipo resol aumenta la estabilidad térmica cuando las concentraciones son superiores al $40 \%$, de lo contrario la estabilidad es igual o inferior a la de la propia resina.

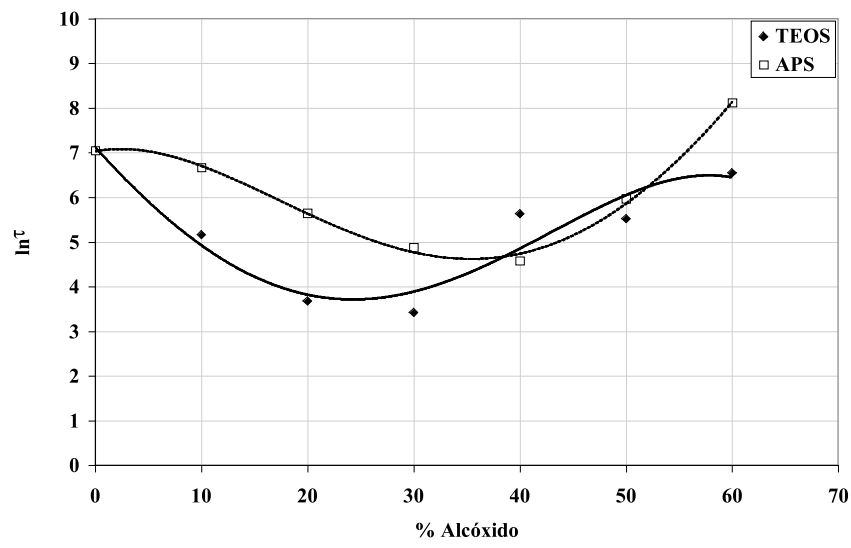

Fig. 11- Tiempo (minutos) de vida media de nanocomposites a una temperatura de $505{ }^{\circ} \mathrm{C}$ y conversión del $95 \%$ (datos ajustados a un polinomio de $4^{\circ}$ orden).

Hasta ahora los resultados obtenidos se han descrito en función de los termogramas TG, sin embargo a continuación se realizará una correlación con los espectros FT-IR. De acuerdo con las Figuras 1 y 2 la incorporación de los alcóxidos TEOS o APS hidrolizados dan lugar a dos tipos de nanoestructuras, una orgánica de resol y otra inorgánica de sílice la cual puede contener los grupos amino-propilo en el caso del APS. En los nanocomposites resol-TEOS los grupos Si-OH del TEOS hidrolizado rompen los enlaces C-O-C que unen los grupos fenólicos, o mejor dicho impiden que se formen tales enlaces, ya que el TEOS hidrolizado se adiciona antes de que polimerice la resina, y mientras que la red no es moyoritariamente inorgánica (es decir para altas concentraciones de resol) la estabilidad térmica es menor que la de la propia resina. Sin embargo a medida que la concentración de TEOS hidrolizado aumenta, la red inorgánica de sílice polimeriza tridimensionalmente y el nanocomposite aumenta en resistencia térmica. En dicha red inorgánica quedarían las moléculas orgánicas del resol.
Por otro lado, en los nanocomposites con APS hidrolizado, para las primeras incorporaciones de APS se produce la rotura de los enlaces $\mathrm{C}-\mathrm{O}-\mathrm{C}$ de forma similar a lo que ocurría con el TEOS hidrolizado, por lo que puede suponerse que son los grupos $\mathrm{Si}-\mathrm{OH}$ los que dan lugar a tales roturas. Este hecho hace que la estabilidad térmica de los nanocomposites con APS disminuya al aumentar el contenido en APS. Sin embargo para altas concentraciones de APS éste polimeriza y forma una red inorgánica similar a la que forma el TEOS hidrolizado pero ahora con estructura lineal (en lugar de tridimensional) y a la vez se deben formar más enlaces C$\mathrm{O}-\mathrm{C}$ a expensas de los $\mathrm{C}-\mathrm{OH}$ del fenol, hecho que debe estar originado por el comportamiento catalítico del APS. Estos dos fenómenos dan lugar a un aumento de la estabilidad térmica del nanocomposite para altos contenidos en APS.

\section{CONCLUSIONES}

De acuerdo a los resultados obtenidos en este estudio se puede concluir que:

a) entre los métodos utilizados (Kissinger, Park y col., Ozawa) para determinar la estabilidad térmica de nanocomposites resol-alcóxido de silicio, el más apropiado es el de Park y col., pues es de gran rapidez y sencillo proporcionando valores tanto de energía de activación como de orden de reacción en todo el intervalo de conversión. Hoy en día el método recomendado por la ASTM es el de Kissinger debido a su relativa sencillez, sin embargo en este trabajo se ha mostrado como el de Park y col., puede ser tan rápido gracias al tratamiento de los datos mediante hojas de cálculo.

b) En cuanto a la degradación térmica de nanocomposites resol-TEOS y resol-APS se ha comprobado que la resistencia disminuye a medida que se aumenta la concentración de alcóxido hasta que ésta alcanza valores comprendidos entre el 30 y $40 \%$. Para concentraciones superiores dicha degradación disminuye (es decir aumenta la estabilidad térmica) con la citada concentración alcanzando resistencias superiores a la de la resina para concentraciones mayores del 50\%. Tanto los valores de energía de activación, IPDT y tiempo de vida media han mostrado tendencias similares.

c) Una explicación relacionada con el comportamiento térmico de los nanocomposites estudiados se ha basado en la espectroscopia FT-IR habiéndose encontrado que para concentraciones inferiores al 40\% los alcóxidos prácticamente no reaccionan con la resina sino que impiden su polimerización, mientras que concentraciones superiores se forma una red inorgánica, ya sea de estructura tridimensional o bien lineal, en la que se encuentra la resina embebida y posiblemente formando enlaces químicos entre la resina y el alcóxido. En el primer caso, es decir para bajas concentraciones de alcóxido, éste debe formar nanopartículas que actúan como puntos de inhomogeneidad en la resina bien impidiendo su polimerización o bien rompiendo enlaces, mientras que en el segundo caso, es decir para altas concentraciones de alcóxido, se forma una red inorgánica-orgánica resistente a la degradación térmica.

\section{AGRADECIMIENTOS}

Este trabajo ha sido realizado dentro de un proyecto financiado por la Comisión Interministerial de Ciencia y 
Tecnología de España (CICYT, Proyecto Ref. MAT2002-03891). Los autores también agradecen a la empresa Fers-Resins la aportación desinteresada de las resina fenólica, así como su uso y aplicación, utilizadas para el fin de este trabajo.

\section{BIBLIOGRAFÍA}

1.- Y. Liu, W-L. Wei, K-Y. Hsu, W-H. Ho. Thermal. Stability of epoxy-silica hybrid materiales by thermogravimetric analysis. Thermochimica Acta . 412, 139-147 (2004).

2.- J-M. Lin, C-C. M. Ma. Thermal degradation of phenolic resin/silica hybrid ceramers. Polymer Degradation and Stability. 69, 229-235 (2000).

3.- Kissinger H. E. Reaction kinetic differential. Anal Chem. 29, 1702-1710 (1957).

4.- T. Ozawa. A new method of analyzing thermogravimetric data. Bull Chem Soc Jpn. 38, 1881-1890 (1965).

5.- H. L. Friedman. Kinetics of thermal degradation of char-forming plastics from thermogravimetry. Application to a phenolic plastic. J. Polym. Sci. PartC . 6, 183-195 (1964)

6.- J. W. Park, S. Cheon Oh, Hae Pyeong Lee, Hee Taik Kim, Kyong Ok Yoo. A kinetic analyisis of thermal degradation of polymers using a dynamic method. Polymer Degradation and Stability. 67, 535-540 (2000).

7.- D. Soriano, A. Mazo, J. Rubio, F. Rubio, J. L. Oteo. Estudio de la Estabilidad Térmica de Nanocomposites Fenólica-TEOS. A.- Actas del VI Congreso Nacional de Materiales Compuestos. AEMAC. Ed. Universidad Politécnica de Valencia. ISBN. 84-9705-821-6. Pp. 353-360, Junio 2005.

8.- N. A. Liu, W.C. Fan. Critical consideration on the Freeman and Carroll meted for evaluating global mass loss kinetics of polymer thermal degradation. Thermochimica Acta. 338, 85-94 (1999).

9.- C. D. Doyle. Estimating thermal stability of experimental polymers by empirical thermogravimetric analysis. Analytical Chemistry. 33, 1, 77-79 (1961).
10.- X.-G. Li, M-R. Huang. Thermal degradation of Kevlar fiber by highresolution thermogravimetry. Journal of Applied Polymer Science. 71, 565-571 (1999).

11.- C. Rockniak, T. Biernacka, M. Skarzynski. Some properties and chemical structure of phenolic resins and their derivatives. J. Appl. Polym. Sci., 28, 531-542 (1983)

12.- L. Costa, L. R. Montelera, G. Camino, E. D. Weil, E. M. Pearce. Structurecharring relationship in phenol-formaldehyde type resins. Polymer Degradation and Stability. 56, 23-35 (1997).

13.- L. Téllez, J. Rubio, E. Morales, F. Rubio, J. L. Oteo. "FT-IR spectroscopy study of the hydrolysis and polycondensation of TEOS-TBT-PDMS." Spectroscopy Letters. 37 (1), 11-31 (2004)

14.- F. Rubio, J. Rubio and J.L. Oteo. A FT-IR Study of the Hydrolysis of Tetaethylortosilicate (TEOS). Spectroscopy Letters, 31 (1), 199-219 (1998)

15.- R. Peña-Alonso, J. Rubio, F. Rubio, J. L. Oteo. Study of the hydrolysis and condensation of $\gamma$-aminopropyltriethoxysilane by FT-IR spectroscopy. J. Mater. Sci. Aceptado para publicar. 2006

16.- K. Hultzsch. Basic reactors in phenolic resin formation. Die Angewandte Chemie A, 60, 179-184 (1948)

17.- J. H. Chan, S. T. Balke. The thermal degradation kinetics of polypropylene: Part III. Thermogravimetric analyses. Polymer Degradation and Stability. 57, 135-149 (1997)

18.- J. Gao, Y. Liu, L. Yang. Thermal stability of boron-containing phenol formaldehyde resin. Polymer Degradation and Stability. 63, 19-22 (1999).

19.- S-J. Park, K-S. Cho. Filler-elastomer interactions: influence of silane coupling agent on crosslink density and thermal stability of silica/rubber composites. Journal of Colloid and Interface Science. 267, 86-91 (2003).

20.- G. P. Wang, T. C. Chang, Y. S. Hong, Y. S. Chiu, C. T. Liu. The effect of silica and siloxane on thermo-oxidative degradation of hydrogen-bonded acidic fluorinated poly(amide-imide-silica) hybrids. Polymer Degradation and Stability. 78, 191-199 (2002).

21.- R. Sengupta, A. Bandyopadhyay, S. Sabharwal, Tapan K. Chaki, Anil K. Bhowmick. Polyamide-6,6/in situ silica hybrid nanocomposites by sol-gel technique: synthesis, characterization and properties. Polymer $46,3343-$ 3354 (2005).

22.- S-J. Park, M-S. Cho. Thermal stability of carbon-MoSi2-carbon composites by thermogravimetric analysis. J. M ater. Sci. 35, 3525-3527 (2000).

Recibido: 23.11.05

Aceptado: 11.08 .06 